\title{
Lernen und Migration
}

\author{
Sarah Widany
}

Online publiziert: 4. März 2015

(c) Die Autor(en) 2015. Dieser Artikel ist auf Springerlink.com mit Open Access verfügbar.

\section{Rezension zu:}

Heinemann, Alisha M. B. (2014). Teilnahme an Weiterbildung in der Migrationsgesellschaft. Perspektiven deutscher Frauen mit „,Migrationshintergrund“. Transcript Verlag, Bielefeld, 328 Seiten, 34,99 €, ISBN 978-3-8376-2718-3

Migration ist in Deutschland schon lange keine Ausnahme, sondern der Normalfall, zumindest mit Blick auf die demographische Entwicklung. Obgleich für die Erwachsenenbildung zahlreiche Bezugspunkte bestehen, liegen bisher nur wenige Publikationen zu migrationsspezifischen Fragestellungen vor.

In dieser Arbeit stehen Frauen mit einem sogenannten Migrationshintergrund im Mittelpunkt. Im Sinne der Adressatenforschung geht es um die Analyse ihrer Beweggründe für und ihrer Perspektiven auf die (Nicht-)Teilnahme an Weiterbildung. Die Schnittstellen zur Migrationsforschung bedient die Autorin durch den systematischen Bezug auf Befunde und theoretische Ansätze aus diesem Kontext. Dadurch wird zunächst aufgezeigt, dass die mit einem „Migrationshintergrund“ verbundenen Segregationslinien in der deutschen Gesellschaft zu Rahmenbedingungen führen, die in einem engen Zusammenhang mit Barrieren der Weiterbildungsteilnahme stehen (S. 47). Teilnahmechancen sind unter diesen Gesichtspunkten verbunden mit Fragen der politischen, ökonomischen, sozialen und kulturellen Teilhabe und den Strukturen und Praktiken, die diese verhindern, wobei hier auch explizit rassistische Praxen adressiert werden. Theoretische Bezugspunkte bilden die in den kultursoziologischen Arbeiten von Pierre Bourdieu entwickelten Konzepte zur Analyse von Machtverhältnissen. Eine Erweiterung erfahren diese durch eine Auswahl postkolonialer Theorieansätze, die es erlauben, das Thema Migration stärker historisch und global zu kontextualisieren (S. 105).

Dr. S. Widany $(\square)$

Freie Universität Berlin,

Berlin, Deutschland

E-Mail: sarah.widany@fu-berlin.de 
Als Gegenüberstellung zu den bisher eher quantitativ orientierten Untersuchungen zum Thema Migration und Weiterbildungsteilnahme wählt die Autorin mit der Grounded Theory und qualitativen Interviews einen interpretativen Zugang. Damit ist auch ein politischer Anspruch verbunden. Einerseits erhalten die Erfahrungen von marginalisierten Gruppen so einen Raum innerhalb des wissenschaftlichen Diskurses (S. 109, 138). Andererseits positioniert sich die Autorin, indem sie die rassismuskritische Forschung als politische Praxis versteht, die die Veränderungen von gesellschaftlichen Verhältnissen intendiert (S. 120). Vor diesem Hintergrund erscheint der Forschungsansatz folgerichtig: Während der methodische Zugang stark subjektbezogen ist, soll der theoretische Bezug eine Objektivierung ermöglichen, indem gesellschaftlich-strukturelle Bedingungen in ihrer Bedeutung für subjektives Handeln beleuchtet werden (S. 137 f.).

Die Auswertung basiert auf Einzelinterviews mit elf Frauen, die ihren Lebensmittelpunkt in Deutschland sehen und einen sogenannten Migrationshintergrund haben. Das relativ kleine Sample veranschaulicht, wie viel Heterogenität eine Gruppe mit diesen Merkmalen mit sich bringen kann, sei es bezüglich des Aufenthaltsstatus, des Bildungsniveaus oder der Deutschkenntnisse. Trotz der beachtlichen Heterogenität werden in der Auswertung verschiedene Dimensionen identifiziert, die in ihren Konstellationen einen bedeutenden Einfluss auf subjektive Weiterbildungsentscheidungen haben. Die wahrgenommene Zugehörigkeit zur deutschen Gesellschaft stellt sich dabei als die zentrale Schlüsseldimension heraus. Abhängig von dem subjektiven Erleben von Zugehörigkeit oder Ausgrenzung nehmen sich die interviewten Frauen als unterschiedlich handlungsfähig wahr und bewerten dementsprechend die Handlungsmöglichkeiten, die ihnen eine Weiterbildungsteilnahme bietet. Entsprechend des zu Beginn formulierten politischen Anspruchs folgen auf Grundlage der Befunde konkrete Empfehlungen und Denkanstöße für die professionelle Arbeit in Weiterbildungseinrichtungen bezüglich der Angebotsgestaltung (S. 283) und für eine kritischreflexive Selbstvergewisserung der pädagogischen Haltung der in der Weiterbildung Tätigen und Forschenden (S. 292).

Praktiker/innen und Forscher/innen empfiehlt sich für die gewinnbringende Lektüre die Bereitschaft, eigene Denkmuster und Privilegien zu reflektieren und zu hinterfragen. Dazu kann die Auseinandersetzung mit den in der Weiterbildungsforschung bisher nicht rezipierten Ansätzen aus dem Bereich der postkolonialen Theorien beitragen, wie auch die ebenso gut aufbereiteten Informationen zu den rechtlichen Rahmenbedingungen für Personen ohne deutsche Staatsangehörigkeit. Angesichts des sehr heterogenen Samples und einer weiten Definition von Weiterbildungsteilnahme stellt sich die Frage, ob eine spezifischere Fragestellung und damit differenziertere Befunde nicht zielführender gewesen wären. Jedoch muss hier dem explorativen Charakter der Untersuchung Rechnung getragen werden. Nicht zuletzt bieten die Ergebnisse, wie Alisha Heinemann in ihren Reflexionen aufzeigt (S. 300), Anschlussmöglichkeiten für weitere Forschungsvorhaben an der Schnittstelle von Weiterbildung und Migration, bspw. mit Blick auf die statistische Erfassung von „Migrationshintergründen“ oder auf einen möglicherweise problematischen Dualismus von Ansprache und Zuschreibung bei Weiterbildungsangeboten, die sich an Zielgruppen mit „Migrationshintergrund“ richten. 
Open Access Dieser Artikel unterliegt den Bedingungen der Creative Commons Attribution License. Dadurch sind die Nutzung, Verteilung, und Reproduktion erlaubt, sofern der/die Originalautor/en und die Quelle angegeben sind. 